\title{
Conjugated Polymer Hydrogel Photocatalysts with Expandable Photoactive Sites in Water
}

\author{
Jeehye Byun, ${ }^{\dagger,+}$ Katharina Landfester, ${ }^{\dagger}{ }^{\dagger}$ and Kai A. I. Zhang ${ }^{*} \dagger^{\dagger}$ \\ ${ }^{\dagger}$ Max Planck Institute for Polymer Research, Ackermannweg 10, 55128 Mainz, Germany \\ ${ }^{\ddagger}$ Water Cycle Research Center, Korea Institute of Science and Technology, Hwarangno 14-gil 5, Seongbuk-gu, Seoul 02792, \\ Republic of Korea
}

Supporting Information

\begin{abstract}
Water compatibility of heterogeneous photocatalytic materials is recently realized by utilizing hydrogels as a support; however, the embedment of photocatalysts in the hydrogels may hinder the photocatalytic activity and the regeneration of materials. Therefore, combining hydrophilicity and high accessibility to the active sites of heterogeneous photocatalysts is highly challenging. Herein, we report the design of a conjugated polymer hydrogel photocatalyst via complexation between a photoactive conjugated polymer with cationic side chains and a poly(acrylic acid) as polyanion. In water, the conjugated polymer hydrogel photocatalyst exhibited an enormous volumetric increase by swelling, which creates more accessible photoactive sites through the simultaneous expansion of the conjugated polymer chain. A simple solvent switch allows the reversible swelling of the hydrogel photocatalyst for recycling. The significantly enhanced photocatalytic activity of the hydrogel photocatalyst was demonstrated by the photodegradation of organic dyes and the formation of the enzyme cofactor nicotinamide adenine dinucleotide via photo-oxidation in water. Furthermore, the translucent and voluminous hydrogel photocatalyst was suitable to pack a large-scale flow column photoreactor for the rapid photocatalytic reactions.
\end{abstract}

\section{INTRODUCTION}

Conjugated polymers have been intensively utilized for heterogeneous photocatalysis under visible light illumination with a strong advantage of structural designability and stability over conventional molecular photocatalysts. ${ }^{1}$ Water-compatible conjugated polymers, in particular, have drawn much attention for their potential use in energy, environmental, and biological applications, namely, water splitting, ${ }^{2-10}$ aqueous pollutant remediation, ${ }^{11-14}$ photodynamic therapy, ${ }^{15-18}$ enzyme regeneration, ${ }^{19-21}$ chemical redox reactions, ${ }^{22-25}$ etc. The hydrophilic conjugated polymer photocatalysts are at present synthesized by surface functionalization, ${ }^{12,26}$ chemical treatment, ${ }^{11}$ and transformation into nanoscale, ${ }^{7,27}$ where the resulting polymers often suffer from difficulty in regeneration due to the irreversible wettability change. Our group recently reported a $\mathrm{CO}_{2}$-responsive hydrophilicity control of conjugated polymer, showing the reversible change of wettability for photoredox reactions in water. ${ }^{28}$

A conventional approach of endowing photocatalysts with water compatibility is to utilize hydrogels as a support. Hydrophilic hydrogel networks can hold huge amount of water by structural swelling, and such a volume change provides inner channels to accommodate guest structures. ${ }^{29-31}$ In this regard, the photocatalysts are loaded on the hydrogel materials to make water-compatible heterogeneous photocatalytic systems. Recent examples include hydrogels with immobilized titanium oxide $\left(\mathrm{TiO}_{2}\right),{ }^{32-36} \mathrm{CdS},{ }^{37}$ porphyrin, ${ }^{38}$ carbon nitride, ${ }^{39,40}$ graphene hydrogels with $\mathrm{TiO}_{2}{ }^{41}$ and selfassembled hydrogel with $\mathrm{Ni}$ catalyst. ${ }^{42}$ The hydrogel photo- catalysts in such composite structures, however, may have to compromise their photocatalytic activity with hydrophilicity, since the photocatalysts are deeply embedded inside the hydrogel networks, limiting the accessibility to photoactive sites. Besides, the three-dimensional hydrogels, albeit easy to be separated, trap the reaction product in the structure, requiring an intensive purification for the regeneration of photocatalysts. ${ }^{34}$

The ideal scenario would be thereby a manipulation of the photocatalytic polymers to have hydrogel-like properties during the catalytic reaction in water, swelling and generating more active sites exposed to the surface. After the reaction, the shrinkage of the polymer network in a poor solvent facilitates the regeneration of the photocatalyst as well as the recovery of the products. If the photoactive conjugated polymer is firmly anchored on another hydrophilic linear polymer via ionic complexation, it can lead to the formation of a threedimensional hydrogel network, ${ }^{43}$ which can repeatedly unfold the conjugated polymer chain by the reversible swelling of network under water uptake. Conjugated polymers containing ionic side chains, namely, conjugated polyelectrolytes are thus a good synthetic platform to achieve the polyelectrolyte hydrogel photocatalyst with expandable photoactive sites, where the properties can be precisely controlled by the choice of polycation and polyanion. ${ }^{44}$

Received: February 7, 2019

Revised: April 2, 2019

Published: April 3, 2019 
In this work, we report the design of conjugated polymer hydrogel photocatalysts via stable ionic complexation between a conjugated polymer containing cationic side chains as the polycation and poly(acrylic acid) (PAA) as the polyanion. The polymer complexes exhibit great water compatibility, absorbing up to 470 times deionized water to its weight. They show large expansion of the photoactive chains while swelling in water. The enhanced photocatalytic activity of the hydrogel photocatalyst was corroborated by the photodegradation of organic dyes and the formation of the enzyme cofactor nicotinamide adenine dinucleotide $\left(\mathrm{NAD}^{+}\right)$via photo-oxidation in water. The hydrogel photocatalyst can be regenerated by a simple solvent exchange after the reaction. The soft, translucent, and voluminous hydrogel photocatalyst can be easily packed in a flow column for a rapid photodegradation of organic dyes with excellent recyclability.

\section{EXPERIMENTAL SECTION}

Materials. 2,7-Dibromo-9,9-bis(6-bromohexyl)fluorine and 2,1,3benzothiadiazole-4,7-diboronic acid pinacol ester were purchased from TCI Germany. Tetrakistriphenylphosphinpalladium(0), XPhos, potassium carbonate, 1-vinylimidazole, and poly(acrylic acid) $\left(M_{\mathrm{w}}\right.$ $250000)$ were bought from Sigma-Aldrich. All the chemicals and solvents were used without purification. Water was used in Millipore quality $(18.2 \mathrm{M} \Omega \mathrm{cm})$.

Synthesis of P-BT-GX $(X=0,9,19,41)$. The polyelectrolyte hydrogel photocatalyst was prepared by the modified method from literature. ${ }^{45}$ Polycation P-BT-Vim $(7 \mathrm{mg}$ ) and polyacrylic acid (PAA, $M_{\mathrm{w}} 250000$ ) were dissolved in dry dimethylformamide (DMF) (10 $\mathrm{mg} \mathrm{mL}^{-1}$ ). (Synthetic details of P-BT-Vim can be found in the Supporting Information.) The amount of PAA was varied to 0, 3, 10.5 , and $63 \mathrm{mg}$ for four P-BT-GX, where $X$ indicates the percentage of PAA remaining in the hydrogel, which was confirmed by thermogravimetric analysis (TGA). The DMF solution was stirred and irradiated under white light-emitting diode (LED) (power: 0.07 $\mathrm{W} \mathrm{cm}{ }^{-2}, \lambda>420 \mathrm{~nm}$ ) overnight, and the DMF was evaporated under low pressure. The mixture was then soaked in 0.5 wt $\% \mathrm{NH}_{4} \mathrm{OH}$ solution overnight to obtain the swollen hydrogel. The crude hydrogel was intensively washed with water and acetone and dried under vacuum at room temperature.

Photocatalytic Degradation of Dye Molecule. P-BT-GX (1 $\mathrm{mg}$ ) was added into rhodamine $\mathrm{B}(\mathrm{RDB})$ dye solution $(10 \mathrm{~mL}, 10 \mathrm{mg}$ $\mathrm{L}^{-1}$ ) and stirred under dark until the adsorption/desorption equilibrium ( $5 \mathrm{~min}$ ). The mixture was exposed under white LED light (power: $0.07 \mathrm{~W} \mathrm{~cm}^{-2}, \lambda>420 \mathrm{~nm}$ ) for the desired time period. Change of dye concentration was monitored through UV-vis spectroscopy. The control experiment with the addition of radical scavengers $(0.5 \mathrm{mmol})$ was conducted under white light irradiation.

Photo-oxidation of NADH Cofactor. P-BT-GX $(2 \mathrm{mg})$ was added into the aqueous solution of $\mathrm{NADH}(20 \mathrm{~mL}, 1 \mathrm{mM})$, and the mixture was purged with $\mathrm{O}_{2}$ for $10 \mathrm{~min}$. The solution was irradiated with white LED (power: $0.07 \mathrm{~W} \mathrm{~cm}{ }^{-2}, \lambda>420 \mathrm{~nm}$ ) in closed condition, and the sample aliquot was taken in regular time intervals. The conversion of $\mathrm{NADH}-\mathrm{NAD}^{+}$was analyzed by $\mathrm{UV}-$ vis spectroscopy, where $\mathrm{NADH}$ showed a signature absorbance peak at $340 \mathrm{~nm}$.

Preparation of a Flow Column Photoreactor Using P-BTG41. The $50 \mathrm{~mL}$ glass column was first packed with glass beads (diameter of $\sim 1 \mathrm{~mm}$ ), and the swollen hydrogel of P-BT-G41 (30 $\mathrm{mg}$ ) was packed on the top. Rhodamine $\mathrm{B}$ dye solution was loaded on the column $\left(10 \mathrm{~mL}, 10 \mathrm{mg} \mathrm{L}^{-1}\right)$ and the column was placed between two white LEDs (power: $0.07 \mathrm{~W} \mathrm{~cm}^{-2}, \lambda>420 \mathrm{~nm}$ ) with the distance of $3 \mathrm{~cm}$. The column was first irradiated with the lights for $3 \mathrm{~min}$ under static condition, then the column valve was open for another 3 min of light exposure during the collection of column effluent. The effluent was analyzed by UV-vis spectroscopy.

Characterization. ${ }^{1} \mathrm{H}$ and ${ }^{13} \mathrm{C}$ NMR spectra were measured by Bruker Avance $300 \mathrm{MHz}$. Solid-state diffuse reflectance (DR) UV-vis spectra were taken from a Perkin-Elmer Lambda 900 UV-vis/NIR spectrometer. UV-vis absorption and emission of liquid samples were monitored by Perkin-Elmer Lambda $25 \mathrm{UV}$-vis spectrometer and J\&M TIDAS spectrofluorometer, respectively. Gel permeation chromatography was performed on a PSS Security of Agilent Technologies 1260 Infinity. Cyclic voltammetry measurement was done using an Autolab PGSTAT204 potentiostat/galvanostat (Metrohm), in which a glassy carbon electrode was a working electrode, $\mathrm{Pt}$ wire was a counter electrode, $\mathrm{Hg} / \mathrm{Hg}_{2} \mathrm{Cl}_{2}$ (in saturated $\mathrm{KCl}$ solution) electrode was a reference electrode, and tetrabutylammonium hexafluorophosphate ( $0.1 \mathrm{M}$ in dimethyl sulfoxide) was the electrolyte. Fourier transform infrared (FTIR) measurement was conducted with Bruker Tensor II FTIR spectrometer. Optical and fluorescence microscopy were carried out on an inverted fluorescence microscope Leica DMi8. Images were recorded on bright field and green fluorescent protein-fluorescein isothiocyanate filter $(485 \mathrm{~nm})$. Thermogravimetric analysis (TGA) was conducted under $\mathrm{N}_{2}$ atmosphere, with temperature ranging from 25 to $800{ }^{\circ} \mathrm{C}$ at a rate of $10^{\circ} \mathrm{C} \mathrm{min}{ }^{-1}$. Static contact angle of polymer deposited on a glass plate was measured using a device equipped with a side camera (IDS uEye camera) and a goniometer. $\zeta$-Potential measurement was carried out with Malvern Zetasizer nanoseries, and aqueous $\mathrm{KCl}$ solution (1 $\mathrm{mM}$ ) was used to dilute the samples $\left(0.3 \mathrm{mg} \mathrm{mL}^{-1}\right)$. The $\mathrm{N}_{2}$ sorption was measured at $77 \mathrm{~K}$ by Quantachrome Autosorb 1 instruments.

\section{RESULTS AND DISCUSSION}

Expanding photoactive sites of the conjugated polymer hydrogel photocatalyst is illustrated in Figure 1. Due to the

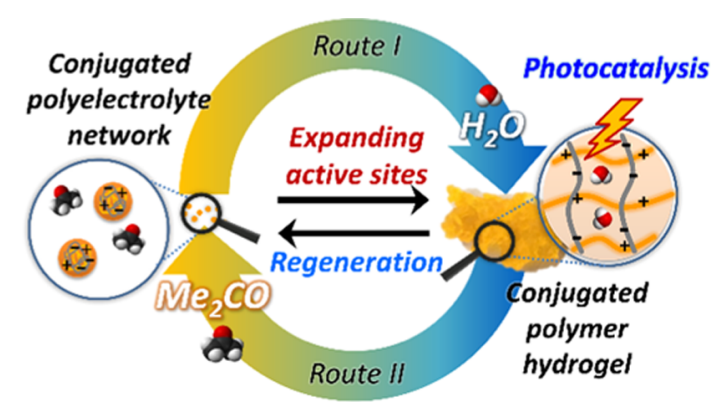

Figure 1. Schematic illustration of the conjugated polymer hydrogel photocatalyst with expandable active sites in water. The swelling of hydrogel photocatalyst was reversible by a solvent exchange.

strong electrostatic interaction between polycation and polyanion, the complex network remained as a small aggregate under dry condition with limited accessibility to its photoactive sites. Once the complex network is added in water (route I in Figure 1), the hydrophilic chain of the polyanion and the ionic groups are hydrated, inducing repulsion between the charges and thereby osmotic pressure to make the complex swollen as being a hydrogel. Since the conjugated polymers with cationic side chains are cross-linked within the complex, the aggregated chains are elongated when the hydrogel swells, leading to the vast expansion of photocatalytic sites exposed for reactions. When the hydrogel is soaked in acetone (route II in Figure 1), on the contrary, acetone replaces the water molecules and dehydrates the hydrogel by the precipitation of hydrophilic polyanion in the poor solvent, with the possible product of the photocatalytic reaction being released for regeneration.

The polyelectrolyte complex was synthesized via modified methods from literature ${ }^{45}$ by using the cationic polyelectrolyte photocatalyst that we reported earlier ${ }^{12}$ (Figure S1). As shown in Figure 2a, the physical blending of the photoactive polycation (P-BT-Vim) and the hydrophilic polyanion (poly- 


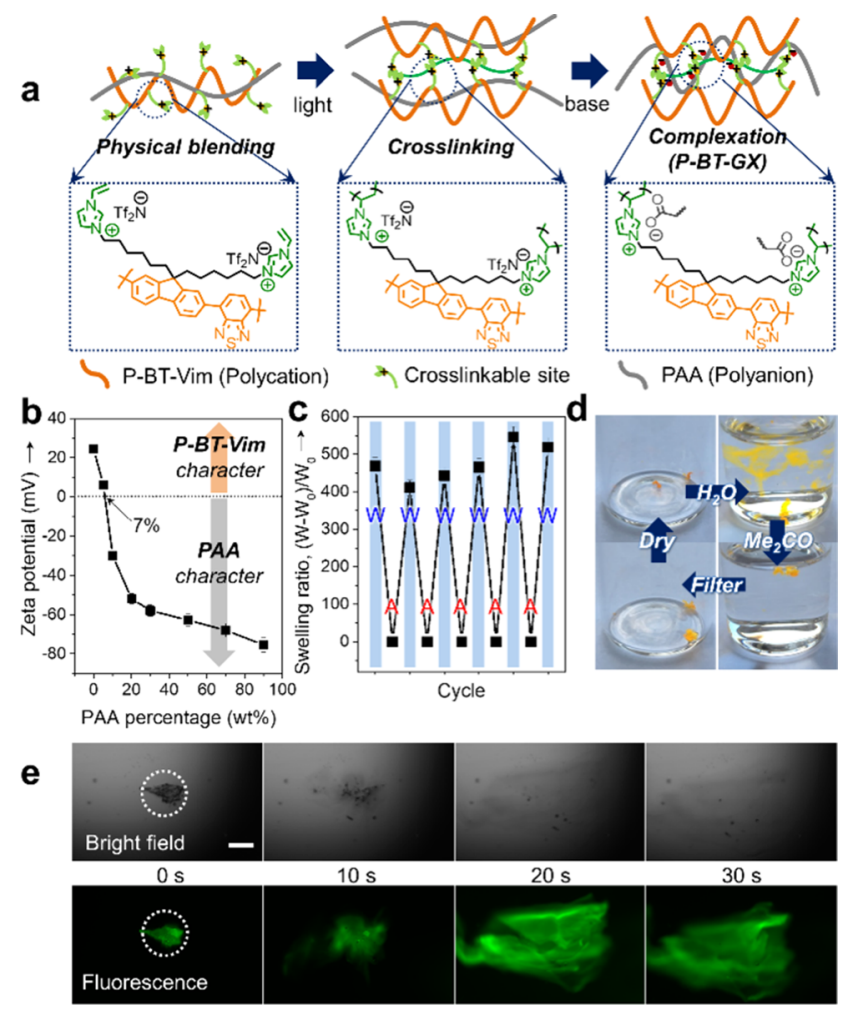

Figure 2. (a) Schematic illustration of synthetic procedure to make conjugated polymer hydrogel photocatalysts, P-BT-GX. (b) $\zeta$ Potential measurement of polyelectrolyte complexes using P-BTVim and PAA in different ratios dispersed in 0.5 wt $\% \mathrm{NH}_{4} \mathrm{OH}$ aqueous solution. For water dispersity, the anion of P-BT-Vim was exchanged to bromide. (c) Reversible swelling behavior of P-BT-G41 by the exchange of water $(\mathrm{W})$ to acetone $(\mathrm{A})$ and $(\mathrm{d})$ photograph of P-BT-G41 during the swelling/deswelling cycle. (e) Optical microscopy images of P-BT-G41 by the addition of water droplet; (top) bright field and (bottom) fluorescence images with excitation wavelength of $485 \mathrm{~nm}$. The swelling behavior was monitored in a regular time trace of $10 \mathrm{~s}$. Scale bar $=0.5 \mathrm{~mm}$.

(acrylic acid), PAA) was first irradiated under visible light for cross-linking the vinylimidazolium units on polycation via selfinitiation (Figure S2 for ${ }^{1} \mathrm{H}$ NMR spectra showing the lightinduced cross-linking process). The mixture was further treated with aqueous $\mathrm{NH}_{4} \mathrm{OH}$ solution for the deprotonation of the carboxylic acid groups to obtain a polyanion, triggering the ionic complexation between polycation and polyanion. The amount of polyanion was varied to be $0,30,60$, and $90 \%$ during the synthesis, resulting in a series of hydrogel photocatalysts, P-BT-GX, where $X$ stands for the weight percentage of polyanion in the hydrogel. Thermogravimetric analysis revealed the actual amount of PAA within the obtained hydrogels to be $0,8.7,19.2$, and $40.7 \%$, thus making P-BT-G0, P-BT-G9, P-BT-G19, and P-BT-G41, respectively (Figure S3). The less amount of PAA remained in the hydrogel compared to the injected amount, indicating that the excess amount of unbound PAA was removed during the washing of hydrogels after the complexation with cross-linked P-BT-Vim. The obtained P-BT-GX were stable in water and other common organic solvents (Figure S4), which is commonly observed in polyelectrolyte complexes. ${ }^{46}$ Among the highly polar solvents, only water allowed the structural swelling of P-BT-GX (Figure S5), since the large dielectric constant of water weakened the Coulombic interaction between polycation and polyanion. ${ }^{47}$
The cross-linking of vinyl units on polycation is thus essential not to excessively loosen the interaction between the polyelectrolytes during the swelling of hydrogel, otherwise it may yield the mass loss of unbound polyanion over cycles.

The FTIR spectroscopy (Figure S6) showed the presence of the deprotonated carboxylate groups in P-BT-GX, with the stretching vibration at $1550 \mathrm{~cm}^{-1}$, which is responsible for the ionic complexation with the polycation. $\zeta$-Potential measurement demonstrated that a one-to-one complexation between the linear P-BT-Vim and PAA was acquired when the weight percentage of the polyanion was ca. $7 \%$ in the polyelectrolyte complexes (Figure 2b), thus P-BT-G19 and P-BT-G41 contained free PAA in the cross-linked networks. According to the $\zeta$-potential profile, P-BT-G0 is supposed to be positively charged, whereas P-BT-G19 and P-BT-G41 are negatively charged in water. P-BT-G9 would be in a neutral state, as it is close to the one-to-one complexation ratio of polyelectrolytes. The surface charge of P-BT-GX may induce a selective interaction toward target substrates. ${ }^{48}$

The swelling ratio of the hydrogel then differed with respect to the amount of the hydrophilic polyanion in the complex (Figure S7). The larger amount of polyanion led to the higher swelling ratio of the hydrogel in water, giving 355 and 470 times higher weight change for P-BT-G19 and P-BT-G41, respectively, which is comparable to conventional PAA-based hydrogels ${ }^{49,50}$ or other derivatives as poly(sulfonic acid) based hydrogels. $^{51}$ With P-BT-G0 and P-BT-G9, the volumetric change was insignificant, likely due to the tight complexation of the polyanion within the larger amount of the cross-linked polycation, as shown in the $\zeta$-potential profile. When the hydrogels were soaked in aqueous $\mathrm{NaCl}$ solution instead, the swelling ratio of P-BT-GX gradually decreased, since the increasing concentration outside of the gel lowered the osmotic pressure for swelling (Figure S7). P-BT-G41 with the highest swelling ratio among the P-BT-GX series, in particular, showed a reversible swelling behavior over several cycles by a solvent exchange, indicating the stability of the hydrogel photocatalyst (Figure 2c). The drastic volumetric change of the hydrogel photocatalyst would allow the effective photocatalytic reactions in water and the isolation of photocatalyst in acetone after the reaction (Figure $2 \mathrm{~d}$ ). We note that the polar solvents with high dielectric constant, such as DMF and acetonitrile, could also dehydrate the swollen hydrogel, ${ }^{52}$ and acetone was suggested as a model solvent due to its high availability and volatility.

In parallel with the swelling of hydrogel, the photoactive conjugated polymer chains were indeed stretched to free more catalytic sites in the material, which was observed by optical microscopy under fluorescent field (Figure 2e). Under the addition of water droplets on dry P-BT-G41, the hydrogel started to swell in a short time period (30 s), and the fluorescent conjugated polymer chain within the hydrogel was also expanded without agglomeration, implying the conjugated polymer became more accessible under water uptake. Freezedried P-BT-G41 after the swelling exhibited the increase in surface area and pore volume compared to the dry P-BT-G41 network, showing $20 \mathrm{~m}^{2} \mathrm{~g}^{-1}$ and $0.098 \mathrm{~m}^{3} \mathrm{~g}^{-1}$ for the freezedried hydrogel and $11.8 \mathrm{~m}^{2} \mathrm{~g}^{-1}$ and $0.083 \mathrm{~m}^{3} \mathrm{~g}^{-1}$ for the dried network, respectively (Figure S8). The improved porosity of the lyophilized hydrogel also supports the better accessibility of conjugated polymer chains to target substrates when in water.

The UV-vis absorption and fluorescent emission spectra of the aqueous dispersion of P-BT-G41 were identical to those of 
the pristine conjugated polymer (P-BT-Vim), retaining the photophysical property even after the ionic complexation with polyanion (Figure S9a). The charge transport within the hydrogel network, however, might be influenced by the presence of excess PAA, as it affects the arrangement of polycations, ${ }^{53}$ which requires future investigation. The UV-vis diffuse reflectance (DR) spectra of P-BT-GX showed a broad absorption in the visible light region up to $550 \mathrm{~nm}$, along with that of the original P-BT-Vim (Figure S9b). The optical band gaps $\left(E_{\mathrm{g}}\right)$ of P-BT-GX and polycation were about $2.2 \mathrm{eV}$, derived from the Kubelka-Munk-transformed reflectance spectra (Figure S9c). According to the reductive potential of P-BT-Vim at $-1.19 \mathrm{~V}$ vs saturated calomel electrode (SCE) obtained by cyclic voltammetry (Figure S9d), the oxidation potentials of P-BT-GX were approximately located at $+1.0 \mathrm{~V}$ vs SCE referring to their optical band gaps.

The effect of the expandable photoactive sites using the conjugated polymer hydrogel photocatalyst has been further demonstrated by photodegradation of water-soluble organic dyes. In a typical experiment, the P-BT-GX series were dispersed in aqueous rhodamine $B(\mathrm{RDB})$ solution in the same concentration and irradiated under white light. As displayed in Figure 3a, P-BT-G0 and P-BT-G9 with minor swelling
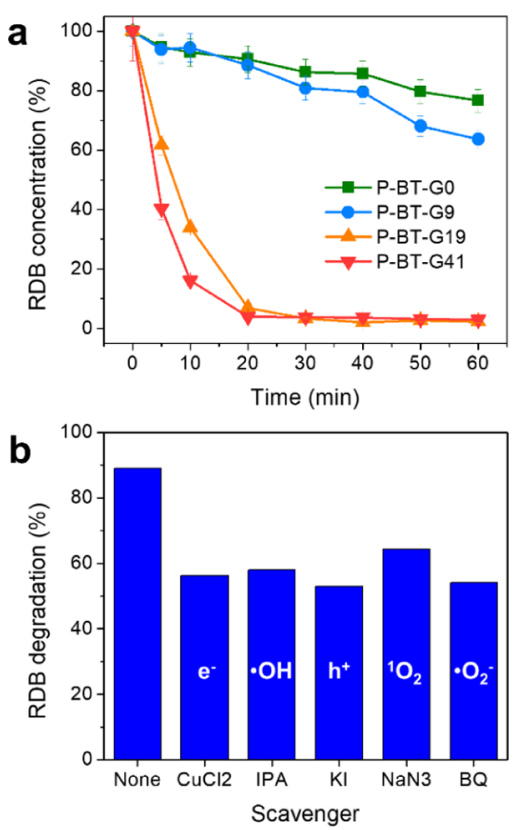

Figure 3. (a) Photocatalytic degradation of rhodamine $B$ (RDB) using P-BT-GX hydrogels. (b) The effect of different scavengers on the RDB degradation over P-BT-G9 for $2 \mathrm{~h}$ of light exposure. The less swelling P-BT-G9 was used to minimize the swelling effect during the photodegradation experiment. Copper(II) chloride $\left(\mathrm{CuCl}_{2}\right)$, isopropanol, potassium iodide (KI), sodium azide $\left(\mathrm{NaN}_{3}\right)$, and 1,4benzoquinone (BQ) were introduced as $\mathrm{e}^{-},{ }^{\bullet} \mathrm{OH}, \mathrm{h}^{+},{ }^{1} \mathrm{O}_{2}$, and ${ }^{\bullet} \mathrm{O}_{2}{ }^{-}$ scavengers, respectively.

properties yielded 23 and 36\% RDB degradation after $1 \mathrm{~h}$ of light irradiation, respectively (see Figure S10 for UV-vis profiles). Despite the less amount of photocatalytic unit, P-BTG9 showed a slight enhancement in degradation efficiency than P-BT-G0, which is attributed to the higher hydrophilicity with the larger amount of polyanion in the structure, corresponding to the smaller contact angle of P-BT-G9 film $\left(72^{\circ}\right)$ than that of P-BT-G0 $\left(85^{\circ}\right)$ (Figure S11). By using P-BT-G19 and P-BT-
G41, the RDB degradation could be completed within 20 min under light illumination. P-BT-G41 having the highest swelling ratio led to the fastest degradation kinetics among the P-BTGX series, even with the smallest content of P-BT-Vim, proving the synergetic effect of water compatibility and expandable photoactive sites of the hydrogel photocatalyst. It is worth noting that the concentration of P-BT-G41 was 10 times less than the conditions of state-of-the-art polymer photocatalysts; ${ }^{13,54}$ moreover, the actual content of the photocatalyst within P-BT-G41 was only 60\% (Figure S3), saving the materials with even higher efficiency. Control experiments in the presence of a series of scavengers revealed that a variety of reactive oxygen species was responsible for the RDB photodegradation, whereas the photogenerated electron/ hole and superoxide radical $\left({ }^{\bullet} \mathrm{O}_{2}^{-}\right)$performed the main role, coinciding with our previous finding ${ }^{28}$ (Figure $3 \mathrm{~b}$ ).

Polyelectrolyte hydrogels are biocompatible due to their hydrophilicity and structural similarity to biological matrix. ${ }^{55}$ The P-BT-GX hydrogel photocatalysts with water compatibility and improved photoactivity thus allowed us to explore the regeneration of the bioactive coenzyme in a photocatalytic way. In living cells, nicotinamide adenine dinucleotide (NAD) exists either in an oxidized form of $\mathrm{NAD}^{+}$or a reduced form of $\mathrm{NADH}$; therefore, the electron transfer through the NAD species involves the oxidation and reduction of organic substrates. The redox reactions of NAD have been investigated by nonenzymatic (photo)chemical approaches, ${ }^{56}$ and the photocatalytic oxidation of $\mathrm{NADH}$ has been much less addressed compared to the reductive regeneration of $\mathrm{NAD}^{+} .{ }^{19,21}$ We then investigated the conversion of $\mathrm{NADH}$ $(1 \mathrm{mM})-\mathrm{NAD}^{+}$under visible light over P-BT-GX, as shown in Figure $4 \mathrm{a}$. The photo-oxidation efficiency of NADH exhibited an increasing trend with respect to the higher swelling ratio of P-BT-GX. P-BT-G19 and P-BT-G41 reached 65 and 99\% of $\mathrm{NADH}$ oxidation in $2 \mathrm{~h}$ of light irradiation, respectively, which

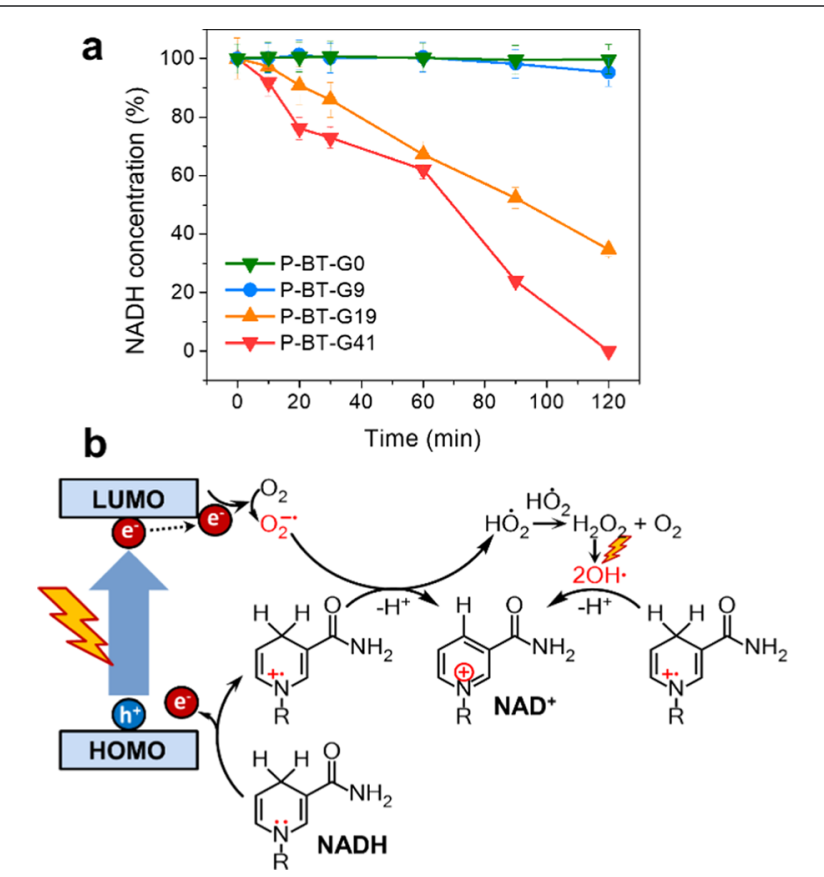

Figure 4. (a) Conversion of NADH to $\mathrm{NAD}^{+}$through photocatalytic oxidation with P-BT-GX hydrogels. (b) Possible mechanism of $\mathrm{NADH}$ oxidation using P-BT-GX as a photocatalyst. 
are well comparable to those of metal-based photocatalysts ${ }^{57}$ (see Figure S12 for UV-vis profiles). In contrast, P-BT-G0 and P-BT-G9 showed only marginal conversion efficiencies owing to the less contact between the relatively hydrophobic photocatalyst and the substrate (Figure S13).

The photo-oxidation of $\mathrm{NADH}$ analogue is known to involve the superoxide, perhydroxyl, and hydroxyl radical species from molecular oxygen; ${ }^{58}$ thus, the control experiment was carried out under oxygen-free condition with P-BT-G41 as a photocatalyst. The conversion efficiency of $\mathrm{NADH}$ was drastically deceased to be $6 \%$ after $2 \mathrm{~h}$ of light exposure, implying the indispensable role of molecular oxygen during the reaction (Figure S12e). Control experiments with a series of radical scavengers further confirmed that hydroxyl and superoxide radicals contributed most to the oxidation of NADH (Figure S14); therefore, the plausible mechanism could be suggested as shown in Figure $4 \mathrm{~b}$. Under light illumination, $\mathrm{NADH}$ was oxidized into the corresponding cationic radical by the photogenerated hole within P-BT-GX. The photoexcited electron in P-BT-GX was quenched by molecular oxygen to form a superoxide radical, where the superoxide radical abstracted a proton from the $\mathrm{NADH}$ cationic radical to give $\mathrm{NAD}^{+}$as a product and perhydroxyl radical as a side product. The accumulated perhydroxyl radical generated peroxide molecules, which could form hydroxyl radical, and the hydroxyl radical readily involved the oxidation of $\mathrm{NAD}^{+}$by capturing proton from the NADH cationic radical.

The large volumetric change of the polymer hydrogel photocatalyst without compromising the photocatalytic activity fits into building a flow column photoreactor. In particular, the swollen P-BT-G41 was highly translucent, making it suitable for a deep light penetration through the column, which overcomes the drawback of polymer photocatalysts in powdery or monolithic textures for the packed column setup. ${ }^{59,60}$ Furthermore, the expanded hydrogel network can provide accessible photocatalytic sites as well as flow channels for target substrate. The hydrated P-BT-G41 could be easily packed in a glass column on the top of glass beads as a spacer (Figure 5a). When loaded with the RDB dye solution and irradiated with visible light for $3 \mathrm{~min}$ under static condition, the hydrogel column quickly degraded the dye, which could be

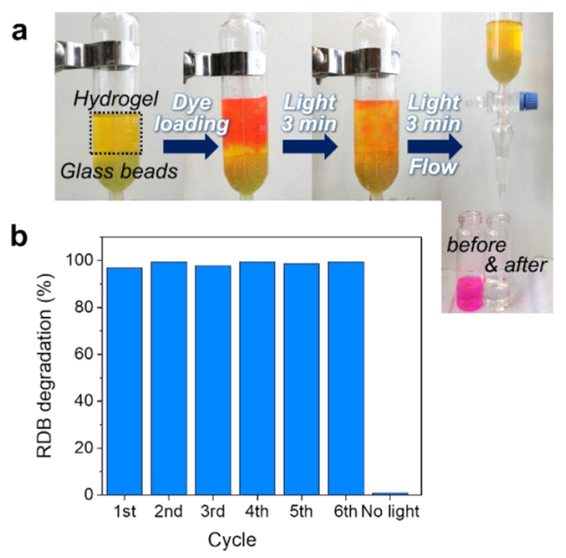

Figure 5. (a) Photographs of a flow column photoreactor packed with P-BT-G41 as a photocatalyst on glass beads. Rhodamine B dye (10 $\mathrm{mL}$ in a cycle) was loaded in the column and irradiated for $3 \mathrm{~min}$. The column was further irradiated for another $3 \mathrm{~min}$ in flow during the collection of the effluent. (b) Recyclability of the column photoreactor during the repeated experiments. observed by its color change. After irradiation for another 3 min in flow, the RDB photodegradation was completed (see video clip in the Supporting Information). In the absence of light as a control, the column photoreactor resulted in only $2 \%$ $\mathrm{RDB}$ removal efficiency, indicating that the adsorption of RDB on the hydrogel was minimal during the $6 \mathrm{~min}$ of column operation. The same RDB photodegradation could be repeated for several cycles without any efficiency loss (Figures $5 b$ and S15). After the cycles, P-BT-G41 exhibited no specific change in FTIR and UV-vis absorption spectra by the virtue of its high stability (Figure S16).

\section{CONCLUSIONS}

In summary, we demonstrated the design of efficient hydrogel photocatalysts via complexation between a photoactive conjugated polymer with cationic side chains and poly(acrylic acid) as a polyanion. The hydrogel photocatalysts exhibited high water compatibility with enormous structural swelling in water, thereby largely expanding the photocatalytic sites. The improved photocatalytic activity was demonstrated by photodegradation of organic dyes and the formation of the enzyme cofactor nicotinamide adenine dinucleotide $\left(\mathrm{NAD}^{+}\right)$via photo-oxidation. The swelling behavior of the hydrogel photocatalysts was reversible by simple solvent exchange, enabling the easy regeneration of the photocatalyst. The highly translucent hydrogel photocatalysts with accessible photoactive sites allowed the construction of a large-scale flow column reactor. We believe our strategy will offer a simple and effective protocol to construct purely organic, water compatible, recyclable, and highly active heterogeneous photocatalysts for a variety of environmentally friendly photocatalytic reactions in aqueous medium.

\section{ASSOCIATED CONTENT}

\section{S Supporting Information}

The Supporting Information is available free of charge on the ACS Publications website at DOI: 10.1021/acs.chemmater.9b00544.

Additional experimental details and characterization such as NMR, TGA, FTIR, $\mathrm{N}_{2}$ sorption isotherm, pore size distribution, UV-vis absorption, cyclic voltammetry, contact angle measurement (PDF)

Flow column photoreactor for rhodamine $\mathrm{b}$ dye degradation (AVI)

\section{AUTHOR INFORMATION}

\section{Corresponding Author}

*E-mail: kai.zhang@mpip-mainz.mpg.de.

ORCID

Katharina Landfester: 0000-0001-9591-4638

Kai A. I. Zhang: 0000-0003-0816-5718

\section{Author Contributions}

The manuscript was written through contributions of all authors. All authors have given approval to the final version of the manuscript.

\section{Notes}

The authors declare no competing financial interest.

\section{ACKNOWLEDGMENTS}

J.B. thanks the Alexander von Humboldt Foundation for the postdoctoral research fellowship (KOR 1188461 HFST-P). 
This work is part of the research conducted by the MaxSynBio consortium that is jointly funded by the Federal Ministry of Education and Research of Germany (BMBF) and the Max Planck Society (MPG) (Grant no. 031A359A MaxSynBio). The authors gratefully thank Dr. Emeline Rideau for microscopy measurement.

\section{REFERENCES}

(1) Zhang, G.; Lan, Z.-A.; Wang, X. Conjugated Polymers: Catalysts for Photocatalytic Hydrogen Evolution. Angew. Chem., Int. Ed. 2016, $55,15712-15727$.

(2) Bi, S.; Lan, Z.-A.; Paasch, S.; Zhang, W.; He, Y.; Zhang, C.; Liu, F.; Wu, D.; Zhuang, X.; Brunner, E.; Wang, X.; Zhang, F. Substantial Cyano-Substituted Fully sp ${ }^{2}$-Carbon-Linked Framework: Metal-Free Approach and Visible-Light-Driven Hydrogen Evolution. Adv. Funct. Mater. 2017, 27, No. 1703146.

(3) Ou, H.; Yang, P.; Lin, L.; Anpo, M.; Wang, X. Carbon Nitride Aerogels for the Photoredox Conversion of Water. Angew. Chem., Int. Ed. 2017, 56, 10905-10910.

(4) Pati, P. B.; Damas, G.; Tian, L.; Fernandes, D. L. A.; Zhang, L.; Pehlivan, I. B.; Edvinsson, T.; Araujo, C. M.; Tian, H. An experimental and theoretical study of an efficient polymer nanophotocatalyst for hydrogen evolution. Energy Environ. Sci. 2017, 10, 1372-1376.

(5) Schwinghammer, K.; Hug, S.; Mesch, M. B.; Senker, J.; Lotsch, B. V. Phenyl-triazine oligomers for light-driven hydrogen evolution. Energy Environ. Sci. 2015, 8, 3345-3353.

(6) Sprick, R. S.; Bonillo, B.; Clowes, R.; Guiglion, P.; Brownbill, N. J.; Slater, B. J.; Blanc, F.; Zwijnenburg, M. A.; Adams, D. J.; Cooper, A. I. Visible-Light-Driven Hydrogen Evolution Using Planarized Conjugated Polymer Photocatalysts. Angew. Chem., Int. Ed. 2016, 55, $1792-1796$.

(7) Wang, L.; Fernández-Terán, R.; Zhang, L.; Fernandes, D. L. A.; Tian, L.; Chen, H.; Tian, H. Organic Polymer Dots as Photocatalysts for Visible Light-Driven Hydrogen Generation. Angew. Chem., Int. Ed. 2016, 55, 12306-12310.

(8) Wang, L.; Wan, Y.; Ding, Y.; Wu, S.; Zhang, Y.; Zhang, X.; Zhang, G.; Xiong, Y.; Wu, X.; Yang, J.; Xu, H. Conjugated Microporous Polymer Nanosheets for Overall Water Splitting Using Visible Light. Adv. Mater. 2017, 29, No. 1702428.

(9) Wang, X.; Maeda, K.; Thomas, A.; Takanabe, K.; Xin, G.; Carlsson, J. M.; Domen, K.; Antonietti, M. A metal-free polymeric photocatalyst for hydrogen production from water under visible light. Nat. Mater. 2009, 8, 76-80.

(10) Zhang, G.; Lin, L.; Li, G.; Zhang, Y.; Savateev, A.; Zafeiratos, S.; Wang, X.; Antonietti, M. Ionothermal Synthesis of TriazineHeptazine-Based Copolymers with Apparent Quantum Yields of 60\% at $420 \mathrm{~nm}$ for Solar Hydrogen Production from "Sea Water". Angew. Chem., Int. Ed. 2018, 57, 9372-9376.

(11) Ghasimi, S.; Landfester, K.; Zhang, K. A. I. Water Compatible Conjugated Microporous Polyazulene Networks as Visible-Light Photocatalysts in Aqueous Medium. ChemCatChem 2016, 8, 694698.

(12) Ghasimi, S.; Prescher, S.; Wang, Z. J.; Landfester, K.; Yuan, J.; Zhang, K. A. I. Heterophase Photocatalysts from Water-Soluble Conjugated Polyelectrolytes: An Example of Self-Initiation under Visible Light. Angew. Chem., Int. Ed. 2015, 54, 14549-14553.

(13) Ghosh, S.; Kouamé, N. A.; Ramos, L.; Remita, S.; Dazzi, A.; Deniset-Besseau, A.; Beaunier, P.; Goubard, F.; Aubert, P.-H.; Remita, $\mathrm{H}$. Conducting polymer nanostructures for photocatalysis under visible light. Nat. Mater. 2015, 14, 505-511.

(14) Lu, C.; Zhang, P.; Jiang, S.; Wu, X.; Song, S.; Zhu, M.; Lou, Z.; Li, Z.; Liu, F.; Liu, Y.; Wang, Y.; Le, Z. Photocatalytic reduction elimination of $\mathrm{UO}_{2}{ }^{2+}$ pollutant under visible light with metal-free sulfur doped $\mathrm{g}_{-} \mathrm{C}_{3} \mathrm{~N}_{4}$ photocatalyst. Appl. Catal., B 2017, 200, 378385.

(15) Geng, J.; Sun, C.; Liu, J.; Liao, L.-D.; Yuan, Y.; Thakor, N.; Wang, J.; Liu, B. Biocompatible Conjugated Polymer Nanoparticles for Efficient Photothermal Tumor Therapy. Small 2015, 11, 16031610.

(16) Xu, L.; Cheng, L.; Wang, C.; Peng, R.; Liu, Z. Conjugated polymers for photothermal therapy of cancer. Polym. Chem. 2014, 5, $1573-1580$.

(17) Yang, K.; Xu, H.; Cheng, L.; Sun, C.; Wang, J.; Liu, Z. In Vitro and In Vivo Near-Infrared Photothermal Therapy of Cancer Using Polypyrrole Organic Nanoparticles. Adv. Mater. 2012, 24, 55865592.

(18) Zhu, C.; Liu, L.; Yang, Q.; Lv, F.; Wang, S. Water-Soluble Conjugated Polymers for Imaging, Diagnosis, and Therapy. Chem. Rev. 2012, 112, 4687-4735.

(19) Liu, J.; Antonietti, M. Bio-inspired NADH regeneration by carbon nitride photocatalysis using diatom templates. Energy Environ. Sci. 2013, 6, 1486-1493.

(20) Liu, J.; Cazelles, R.; Chen, Z. P.; Zhou, H.; Galarneau, A.; Antonietti, M. The bioinspired construction of an ordered carbon nitride array for photocatalytic mediated enzymatic reduction. Phys. Chem. Chem. Phys. 2014, 16, 14699-14705.

(21) Oppelt, K. T.; Gasiorowski, J.; Egbe, D. A. M.; Kollender, J. P.; Himmelsbach, M.; Hassel, A. W.; Sariciftci, N. S.; Knör, G. RhodiumCoordinated Poly(arylene-ethynylene)-alt-Poly(arylene-vinylene) Copolymer Acting as Photocatalyst for Visible-Light-Powered $\mathrm{NAD}^{+}$/ NADH Reduction. J. Am. Chem. Soc. 2014, 136, 12721-12729.

(22) Ayed, C.; Caire da Silva, L.; Wang, D.; Zhang, K. A. I. Designing conjugated microporous polymers for visible lightpromoted photocatalytic carbon-carbon double bond cleavage in aqueous medium. J. Mater. Chem. A 2018, 6, 22145-22151.

(23) Chen, L.; Zhao, L.; Xu, J.; Long, J.; Hou, L. Metal-Free Photocatalysts $\mathrm{C}_{3} \mathrm{~N}_{3} \mathrm{~S}_{3}$ and its Polymers: Solubility in Water and Application in Benzylic Alcohols Oxidation Under Visible Light. Nano 2017, 12, No. 1750101.

(24) Huang, W.; Wang, Z. J.; Ma, B. C.; Ghasimi, S.; Gehrig, D.; Laquai, F.; Landfester, K.; Zhang, K. A. I. Hollow nanoporous covalent triazine frameworks via acid vapor-assisted solid phase synthesis for enhanced visible light photoactivity. J. Mater. Chem. A 2016, 4, 7555-7559.

(25) Long, B.; Ding, Z.; Wang, X. Carbon Nitride for the Selective Oxidation of Aromatic Alcohols in Water under Visible Light. ChemSusChem 2013, 6, 2074-2078.

(26) Urakami, H.; Zhang, K.; Vilela, F. Modification of conjugated microporous poly-benzothiadiazole for photosensitized singlet oxygen generation in water. Chem. Commun. 2013, 49, 2353-2355.

(27) Ma, B. C.; Ghasimi, S.; Landfester, K.; Zhang, K. A. I. Enhanced visible light promoted antibacterial efficiency of conjugated microporous polymer nanoparticles via molecular doping. J. Mater. Chem. B 2016, 4, 5112-5118.

(28) Byun, J.; Huang, W.; Wang, D.; Li, R.; Zhang, K. A. I. $\mathrm{CO}_{2-}$ Triggered Switchable Hydrophilicity of a Heterogeneous Conjugated Polymer Photocatalyst for Enhanced Catalytic Activity in Water. Angew. Chem., Int. Ed. 2018, 57, 2967-2971.

(29) Fu, Q.; Duan, C.; Yan, Z.; Li, Y.; Si, Y.; Liu, L.; Yu, J.; Ding, B. Nanofiber-Based Hydrogels: Controllable Synthesis and Multifunctional Applications. Macromol. Rapid Commun. 2018, 39, No. 1800058.

(30) Jiang, W.; Zhu, Y.; Zhu, G.; Zhang, Z.; Chen, X.; Yao, W. Three-dimensional photocatalysts with a network structure. J. Mater. Chem. A 2017, 5, 5661-5679.

(31) Zhao, F.; Bae, J.; Zhou, X.; Guo, Y.; Yu, G. Nanostructured Functional Hydrogels as an Emerging Platform for Advanced Energy Technologies. Adv. Mater. 2018, 30, No. 1801796.

(32) Badranova, G. U.; Gotovtsev, P. M.; Zubavichus, Y. V.; Staroselskiy, I. A.; Vasiliev, A. L.; Trunkin, I. N.; Fedorov, M. V. Biopolymer-based hydrogels for encapsulation of photocatalytic $\mathrm{TiO}_{2}$ nanoparticles prepared by the freezing/thawing method. J. Mol. Liq. 2016, 223, 16-20.

(33) Kazemi, F.; Mohamadnia, Z.; Kaboudin, B.; Karimi, Z. Photodegradation of methylene blue with a titanium dioxide/ 
polyacrylamide photocatalyst under sunlight. J. Appl. Polym. Sci. 2016, 133, No. 43386

(34) Mai, N. X. D.; Bae, J.; Kim, I. T.; Park, S. H.; Lee, G.-W.; Kim, J. H.; Lee, D.; Son, H. B.; Lee, Y.-C.; Hur, J. A recyclable, recoverable, and reformable hydrogel-based smart photocatalyst. Environ. Sci. Nano 2017, 4, 955-966.

(35) Mai, N. X. D.; Park, D.; Yoon, J.; Hur, J. Comparative Study of Hydrogel-Based Recyclable Photocatalysts. J. Nanosci. Nanotechnol. 2018, 18, 1361-1364.

(36) Harikumar, P. S.; Joseph, L.; Dhanya, A. Photocatalytic degradation of textile dyes by hydrogel supported titanium dioxide nanoparticles. J. Environ. Eng. Ecol. Sci. 2013, 2, 2.

(37) Yang, J.; Gao, J.; Wang, X.; Mei, S.; Zhao, R.; Hao, C.; Wu, Y.; Zhai, X.; Liu, Y. Polyacrylamide hydrogel as a template in situ synthesis of CdS nanoparticles with high photocatalytic activity and photostability. J. Nanopart. Res. 2017, 19, 350.

(38) Frühbeißer, S.; Mariani, G.; Gröhn, F. Porphyrin DiacidPolyelectrolyte Assemblies: Effective Photocatalysts in Solution. Polymers 2016, 8, No. 180.

(39) Liu, J.; An, T.; Chen, Z.; Wang, Z.; Zhou, H.; Fan, T.; Zhang, D.; Antonietti, M. Carbon nitride nanosheets as visible light photocatalytic initiators and crosslinkers for hydrogels with thermoresponsive turbidity. J. Mater. Chem. A 2017, 5, 8933-8938.

(40) Sun, J.; Schmidt, B. V. K. J.; Wang, X.; Shalom, M. SelfStanding Carbon Nitride-Based Hydrogels with High Photocatalytic Activity. ACS Appl. Mater. Interfaces 2017, 9, 2029-2034.

(41) Castillo, V. C.; Dalagan, J. Q. Graphene/ $\mathrm{TiO}_{2}$ hydrogel: a potential catalyst to hydrogen evolution reaction. Bull. Mater. Sci. 2016, 39, 1461-1466.

(42) Weingarten, A. S.; Kazantsev, R. V.; Palmer, L. C.; McClendon, M.; Koltonow, A. R.; Samuel, A. P. S.; Kiebala, D. J.; Wasielewski, M. R.; Stupp, S. I. Self-assembling hydrogel scaffolds for photocatalytic hydrogen production. Nat. Chem. 2014, 6, 964-970.

(43) Voorhaar, L.; Hoogenboom, R. Supramolecular polymer networks: hydrogels and bulk materials. Chem. Soc. Rev. 2016, 45, 4013-4031.

(44) Jiang, X.-S.; Mathew, M. P.; Du, J. Polyelectrolyte Hydrogels: Thermodynamics. In Polyelectrolytes: Thermodynamics and Rheology; Visakh, P. M.; Bayraktar, O.; Picó, G. A., Eds.; Springer International Publishing: Cham, 2014; pp 183-214.

(45) Zhao, Q.; Yin, M.; Zhang, A. P.; Prescher, S.; Antonietti, M.; Yuan, J. Hierarchically Structured Nanoporous Poly(Ionic Liquid) Membranes: Facile Preparation and Application in Fiber-Optic pH Sensing. J. Am. Chem. Soc. 2013, 135, 5549-5552.

(46) Thünemann, A. F.; Müller, M.; Dautzenberg, H.; Joanny, J.-F.; Löwen, H. Polyelectrolyte Complexes. In Polyelectrolytes with Defined Molecular Architecture II; Schmidt, M., Ed.; Springer: Berlin, 2004; pp 113-171.

(47) Muthukumar, M. 50th Anniversary Perspective: A Perspective on Polyelectrolyte Solutions. Macromolecules 2017, 50, 9528-9560.

(48) Byun, J.; Patel, H. A.; Thirion, D.; Yavuz, C. T. Charge-specific size-dependent separation of water-soluble organic molecules by fluorinated nanoporous networks. Nat. Commun. 2016, 7, No. 13377.

(49) Li, A.; Wang, A.; Chen, J. Studies on poly(acrylic acid)/ attapulgite superabsorbent composite. I. Synthesis and characterization. J. Appl. Polym. Sci. 2004, 92, 1596-1603.

(50) Mo, C.; Shu-quan, Z.; Hua-Min, L.; Zhan-bin, H.; Shu-qin, L. Synthesis of poly(acrylic acid)/sodium humate superabsorbent composite for agricultural use. J. Appl. Polym. Sci. 2006, 102, 51375143.

(51) Kovačič, S.; Silverstein, M. S. Superabsorbent, High Porosity, PAMPS-Based Hydrogels through Emulsion Templating. Macromol. Rapid Commun. 2016, 37, 1814-1819.

(52) Nishiyama, Y.; Satoh, M. Solvent- and counterion-specific swelling behavior of poly(acrylic acid) gels. J. Polym. Sci. B 2000, 38, 2791-2800.

(53) Kim, S.-M.; Kim, C.-H.; Kim, Y.; Kim, N.; Lee, W.-J.; Lee, E.H.; Kim, D.; Park, S.; Lee, K.; Rivnay, J.; Yoon, M.-H. Influence of PEDOT:PSS crystallinity and composition on electrochemical transistor performance and long-term stability. Nat. Commun. 2018, 9, No. 3858.

(54) Shalom, M.; Inal, S.; Fettkenhauer, C.; Neher, D.; Antonietti, M. Improving Carbon Nitride Photocatalysis by Supramolecular Preorganization of Monomers. J. Am. Chem. Soc. 2013, 135, 71187121.

(55) Kwon, H. J.; Yasuda, K.; Gong, J. P.; Ohmiya, Y. Polyelectrolyte hydrogels for replacement and regeneration of biological tissues. Macromol. Res. 2014, 22, 227-235.

(56) Wang, X.; Saba, T.; Yiu, H. H. P.; Howe, R. F.; Anderson, J. A.; Shi, J. Cofactor $\mathrm{NAD}(\mathrm{P}) \mathrm{H}$ Regeneration Inspired by Heterogeneous Pathways. Chem 2017, 2, 621-654.

(57) Lin, S.; Sun, S.; Wang, K.; Shen, K.; Ma, B.; Ren, Y.; Fan, X. Bioinspired Design of Alcohol Dehydrogenase@nano $\mathrm{TiO}_{2}$ Microreactors for Sustainable Cycling of $\mathrm{NAD}^{+} / \mathrm{NADH}$ Coenzyme. Nanomaterials 2018, 8, No. 127.

(58) Fukuzumi, S.; Lee, Y.-M.; Jung, J.; Nam, W. Thermal and photocatalytic oxidation of organic substrates by dioxygen with water as an electron source. Green Chem. 2018, 20, 948-963.

(59) Wang, Z. J.; Ghasimi, S.; Landfester, K.; Zhang, K. A. I. Highly porous conjugated polymers for selective oxidation of organic sulfides under visible light. Chem. Commun. 2014, 50, 8177-8180.

(60) Zhang, K.; Vobecka, Z.; Tauer, K.; Antonietti, M.; Vilela, F. [small pi]-Conjugated polyHIPEs as highly efficient and reusable heterogeneous photosensitizers. Chem. Commun. 2013, 49, 1115811160 . 